\title{
SÍNTESE E CARACTERIZAÇÃO DE UMA ARGILA BENTONÍTICA PILARIZADA COM PILARES MISTOS DE Al/Fe E IMPREGNADA COM Pd
}

\author{
M. V. C. SILVA ${ }^{1}$, P. W. T. S. TORRES ${ }^{1}$, J. L. FERREIRA ${ }^{2}$, A. H. PIZARRO ${ }^{3}$ e C. B. \\ MOLINA $^{3}$ \\ ${ }^{1}$ Universidade Federal do Pará, Faculdade de Engenharia Química \\ ${ }^{2}$ Universidade Federal do Pará, Programa de Pós Graduação em Engenharia Química \\ ${ }^{3}$ Universidad Autónoma de Madrid, Sección de Ingeniería Química \\ E-mail para contato: marcus.ufpa@yahoo.com.br
}

\begin{abstract}
RESUMO - Bentonitas pilarizadas com pilares mistos de $\mathrm{Al} / \mathrm{Fe}$ são excelentes catalisadores de reações de oxidação. Quando o Pd é impregnado, esses materiais se tornam catalisadores de reações de hidrodescloração e oxidação ao mesmo tempo, por exemplo. Neste trabalho, uma bentonita norte-americana foi pilarizada com polihidroxicátions de $\mathrm{Al}$ e Fe, gerando a Al-Fe-PILC. Em seguida o Pd foi impregnado ao material, gerando a Pd-Al-Fe-PILC. As amostras foram caracterizadas por DRX, ATD/TG e pela análise de área superficial específica (ASE) pelo método BET. Houve um aumento do espaçamento basal da bentonita de $12,4 \mathrm{~A}^{\circ}$ na amostra original para $17,81 \mathrm{~A}^{\circ}$ na Al-Fe-PILC e $17,05 \mathrm{~A}^{\circ}$ na PdAl-Fe-PILC. A ASE aumentou de $19,05 \mathrm{~m}^{2} / \mathrm{g}$ na amostra original para 173,49 $\mathrm{m}^{2} / \mathrm{g}$ na Al-Fe-PILC e $92 \mathrm{~m}^{2} / \mathrm{g}$ na Pd-Al-Fe-PILC. As estabilidades térmicas dos materiais foram bastante elevadas, com a desidroxilação (colapso parcial da estrutura) ocorrendo em torno de $678^{\circ} \mathrm{C}$. O processo de pilarização ocorreu com sucesso e os materiais sintetizados possuem grande potencial para serem utilizados como catalisadores.
\end{abstract}

\section{INTRODUÇÃO}

Argilas pilarizadas são materiais que possuem porosidade permanente, obtida através da introdução de compostos químicos que funcionam como pilares de dimensão molecular entre as lamelas da argila, mantendo-as afastadas e dando origem aos microporos. Os compostos químicos que funcionam como suportes ou pilares moleculares entre as lamelas da argila são denominados agentes pilarizantes. A simples introdução do agente pilarizante por troca iônica dá origem às argilas intercaladas. $O$ processo de calcinação que se segue elimina a água presente entre as lamelas produzindo a microporosidade característica das argilas pilarizadas (Luna e Schuchardt, 1999).

O processo de preparação de uma argila pilarizada está resumido nos seguintes passos: inchamento da esmectita com água; troca dos cátions interlamelares por complexos metálicos oligoméricos ou poliméricos parcialmente hidratados; secagem e calcinação para transformação dos precursores polioxocátions em pilares de óxido metálico. Nessa última 
etapa, os pilares óxidos se ligam covalentemente às folhas tetraédricas da argila, mantendo-as separadas permanentemente (Teixeira-Neto e Teixeira-Neto, 2009).

Nos últimos anos, argilas pilarizadas têm sido bastante utilizadas como catalisadores de reações químicas, como por exemplo: oxidação de tartazina (Bankovic et al., 2012), síntese de 2,2-dimetil-1,3-dioxolano (Mnasri et al., 2012), pirólise catalítica de polietileno (De Stefanis et al., 2013) e hidroisomerização do heptano (Kooli, 2014).

Argilas pilarizadas com pilares mistos são sintetizadas a fim de produzir materiais com estabilidade térmica mais elevada e para serem utilizadas em reações químicas mais específicas. Ultimamente diversos estudos estão sendo realizados com esses materiais, como por exemplo: Zr-Fe-PILC (Molina et al., 2006) e Fe-Cr-PILC (Tomul, 2012) na oxidação catalítica de fenol, Al-Cu-PILC na oxidação de alaranjado de metila (Galeano et al., 2010), Al-Fe-PILC para oxidação catalítica de 4-clorofenol (Catrinescu et al., 2012) e Cr-Al-PILC na desidrogenação oxidativa de propano (León et al., 2014).

Recentemente está sendo estudada a realização simultânea de reações de oxidação e hidrodescloração de 4-clorofenol, com o objetivo de produzir compostos bem menos contaminantes e evitar a formação de subprodutos tóxicos (Yalfani et al., 2011; Munoz et al., 2013; Silva et al., 2014). Para esses estudos, os autores utilizaram catalisadores que atuam simultaneamente nas duas reações citadas. Dessa forma, o presente trabalho teve como objetivar sintetizar e caracterizar uma argila bentonítica com pilares mistos de $\mathrm{Al} / \mathrm{Fe}$, que é muito utilizada em reações de oxidação. Objetivou-se também sintetizar e caracterizar um material que poderá ser utilizado em reações simultâneas de oxidação e hidrodescloração, a partir da impregnação de paládio na bentonita pilarizada com pilares mistos de $\mathrm{Al} / \mathrm{Fe}$.

\section{MATERIAIS E MÉTODOS}

\subsection{Processo de pilarização com Al/Fe e impregnação de Pd}

A argila bentonítica utilizada nesse trabalho é proveniente da Fisher Scientific Company / USA. Para o processo de pilarização com Al e Fe, a primeira etapa realizada foi a preparação da solução pilarizante, pela adição lenta de $\mathrm{NaOH}(0,2 \mathrm{M})$ a uma solução de $\mathrm{Al}\left(\mathrm{NO}_{3}\right)_{3} \cdot 9 \mathrm{H}_{2} \mathrm{O}$ $(0,1 \mathrm{M})$ misturada com uma solução de $\mathrm{Fe}\left(\mathrm{NO}_{3}\right)_{3} \cdot 9 \mathrm{H}_{2} \mathrm{O}$, sob agitação magnética contínua à temperatura ambiente. A solução resultante foi mantida a uma temperatura de $90^{\circ} \mathrm{C}$ durante 3 horas e posteriormente mantida à temperatura ambiente por duas horas. A solução pilarizante foi adicionada lentamente à suspensão de argila $(1 \% \mathrm{~m} / \mathrm{v})$, sob agitação magnética contínua à temperatura ambiente. Após esse processo de intercalação, a mistura foi lavada sucessivas vezes por agitação/centrifugação até condutividade próxima da água destilada. $\mathrm{O}$ produto sólido obtido foi seco a $60^{\circ} \mathrm{C}$ por 15 horas e calcinado a $350^{\circ} \mathrm{C}$ por duas horas. Em seguida, o material foi desagregado em almofariz, classificado abaixo de $0,15 \mathrm{~mm}$ (100 mesh) em peneiramento manual e recebeu o nome de Al-Fe-PILC. Esse metodologia teve como referência o estudo de Cañizares et al. (1999).

A Al-Fe-PILC sintetizada foi tratada com uma solução de $\mathrm{PdCl}_{2}$ (dissolvido em $\mathrm{HCl}$ $1 \mathrm{M}$ ), pelo método da impregnação (Vicente e Lambert, 2001). Em seguida a amostra foi seca a $25^{\circ} \mathrm{C}$ por duas horas e a $60^{\circ} \mathrm{C}$ durante 15 horas. Posteriormente, a amostra foi calcinada a 
$500^{\circ} \mathrm{C}$ por duas horas. Esse catalisador foi sintetizado com $1 \%$ em massa de $\mathrm{Pd}$ e recebeu o nome de Pd-Al-Fe-PILC.

\subsection{Técnicas de caracterização}

A amostra natural e os materiais sintetizados foram caracterizados por Difração de Raios-X, análise da área superficial específica pelo método BET e Análise Termodiferencial.

Difração de Raios-X: as análises foram realizadas em um Difratômetro de Raios-X, modelo X'PERT PRO MPD (PW 3040/60), da PANalytical, com goniômetro PW3050/60 ( $\theta$ / $\theta)$, tubo de Raios-X cerâmico e anodo de $\mathrm{Cu}(\mathrm{K} \alpha 1=1,540598 \AA$ A), modelo PW3373/00 com foco fino $(2200 \mathrm{~W} / 60 \mathrm{kV})$, e filtro $\mathrm{K} \beta$ de Níquel. As condições instrumentais utilizadas foram as seguintes: varredura de 3 a $75^{\circ}$ em $2 \theta$, voltagem de $40 \mathrm{kV}$, corrente de $30 \mathrm{~mA}$, tamanho do passo de $0,02^{\circ}$ em $2 \theta$, tempo/passo de $81 \mathrm{~s}$, fenda divergente de $1 / 4^{\circ}$ e antiespalhamento de $1 / 2^{\circ}$, máscara de $10 \mathrm{~mm}$ e movimentação circular da amostra com frequência de 1 rotação/s. A aquisição de dados foi feita com o software X'Pert Data Collector, e o tratamento dos dados com o software X’Pert HighScore.

Área superficial específica (ASE): a área superficial específica foi obtida a partir de medidas de adsorção de $\mathrm{N}_{2}$ a $77 \mathrm{~K}$, usando analisador QUANTACHROME/Nova - 1200 . Através dessas medidas foram obtidas as isotermas de adsorção, a partir das quais foram calculados os valores de ASE pelo método BET (Brunauer - Emmett - Teller). Antes das medidas de adsorção, as amostras passaram por pré-tratamento térmico a $200{ }^{\circ} \mathrm{C}$ por $2 \mathrm{~h}$.

Análise Termodiferencial: as análises foram realizadas em um equipamento modelo PL Thermal Science com analisador térmico simultâneo STA1000/1500, da Stanton Redcroft Ltda, forno cilíndrico vertical e conversor digital acoplado a um microcomputador. As amostras foram depositadas em cadinhos de alumina. A varredura iniciou na faixa de temperatura ambiente até $1000^{\circ} \mathrm{C}$, com uma taxa de aquecimento de $20^{\circ} \mathrm{C} / \mathrm{min}$.

\section{RESULTADOS E DISCUSSÃO}

Na bentonita utilizada nesse trabalho há a presença predominante da montmorillonitaesmectita, com a identificação dos seus três picos principais. Comparando os difratogramas da bentonita natural e da Al-Fe-PILC, pode-se verificar que o espaçamento basal - d $(\AA)$ - das

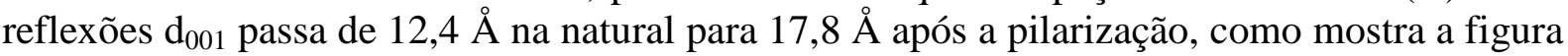
1. $\mathrm{O}$ valor de $\mathrm{d}_{001}$ alcançado para a Al-Fe-PILC é similar aos obtidos por outros trabalhos empregando outras metodologias: Bankovic et al. (2012) alcançaram 17,4 $\AA$, Catrinescu et al.

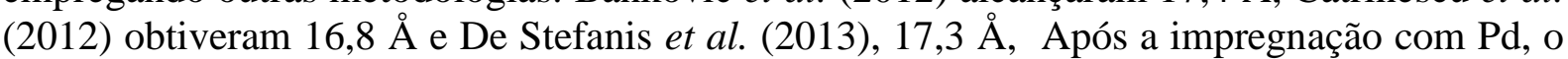
espaçamento permaneceu praticamente constante, 17,7 $\AA$. Esse aumento no espaçamento basal é um indicativo de que os polioxidocátions de $\mathrm{Al}$ e $\mathrm{Fe}$ foram fortemente fixados nos espaços interlamelares da montmorillonita depois da calcinação, comprovando a eficiência do método de pilarização. Na Pd-Al-Fe-PILC, apesar do pico principal da montmorillonita apresentar um valor elevado de espaçamento basal, houve uma grande redução da cristalinidade e da intensidade, mostrando que grande parte dos pilares sofreram colapso, possivelmente devido a alta acidez da solução de $\mathrm{PdCl}_{2}$ utilizada. Esse mesmo comportamento foi obtido por Oliveira et al. (2008). 


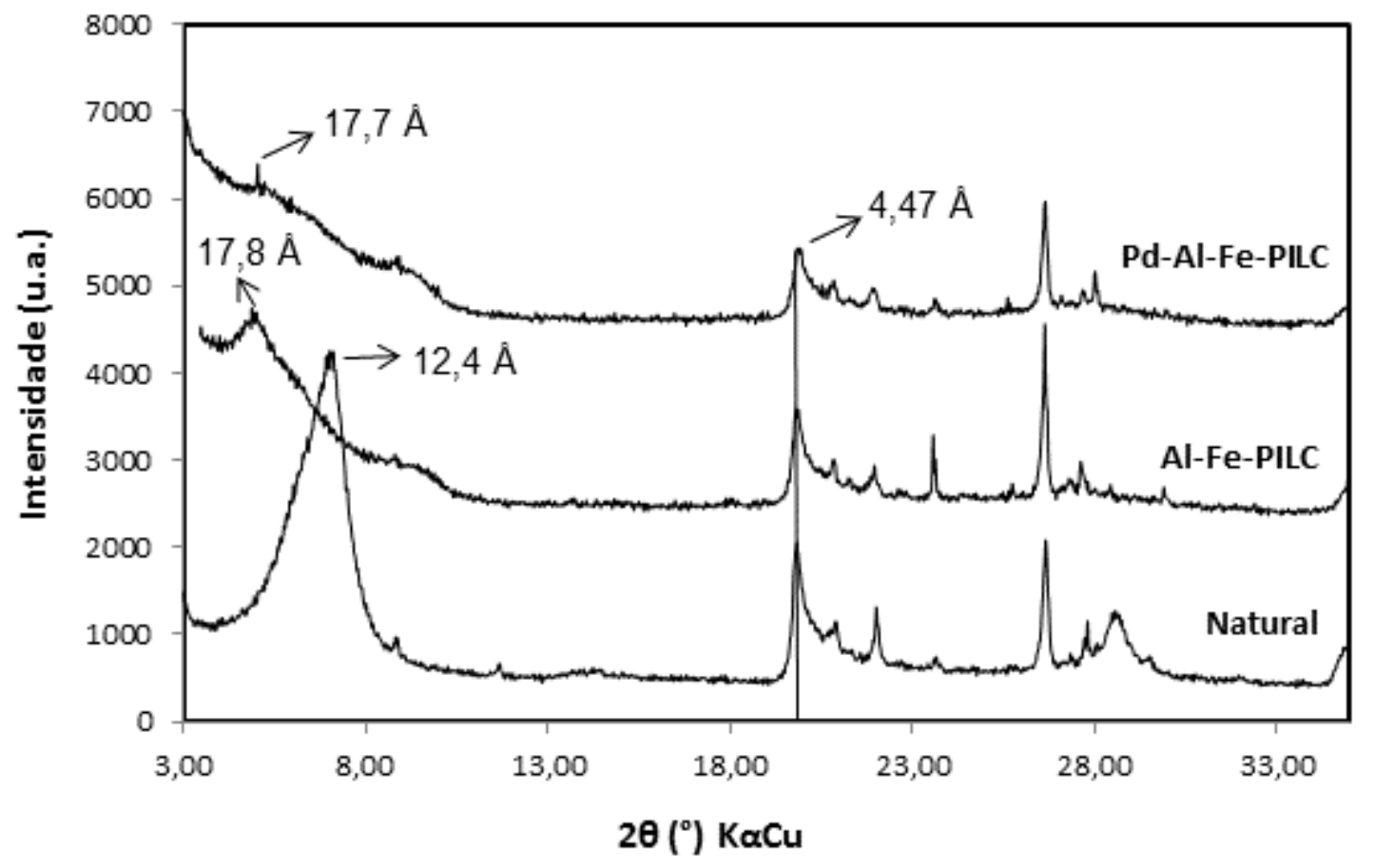

Figura 1 - Difratogramas da amostra natural, Al-Fe-PILC e Pd-Al-Fe-PILC

De acordo com a tabela 1, verifica-se que com a pilarização o valor da área superficial específica (ASE) aumentou em mais de 9 vezes o valor da argila natural, passando de 19,05 a $173,49 \mathrm{~m}^{2} / \mathrm{g}$, comprovando o sucesso do experimento. Essa é uma consequência da criação de microporos entre as lamelas da argila após a pilarização, surgindo uma porosidade regular. $\mathrm{O}$ valor de $173,49 \mathrm{~m}^{2} / \mathrm{g}$ obtido nesse trabalho para a Al-Fe-PILC foi ligeiramente maior do que os alcançados por Bankovic et al. (2012) e Catrinescu et al. (2012), com valores de ASE de $151 \mathrm{~m}^{2} / \mathrm{g}$ e $160 \mathrm{~m}^{2} / \mathrm{g}$, respectivamente. Após a impregnação do Pd, houve uma significativa diminuição da ASE, com um valor de $92 \mathrm{~m}^{2} / \mathrm{g}$. Esse mesmo comportamento foi observado em outros trabalhos, em que após a impregnação de metais como $\mathrm{Pd}, \mathrm{Ce}, \mathrm{Au}, \mathrm{Cr}, \mathrm{Pt}$ e $\mathrm{Rh}$, o valor da ASE diminui. Esse fato possivelmente ocorre devido aos metais se introduzirem nas camadas da argila e se depositarem nos poros (Oliveira et al., 2008; Molina et al., 2009; Alvarez et al., 2012; Molina et al., 2014; Mnasri-Ghnimi et al., 2014).

Tabela 1 - Valores de Área Superficial Específica (ASE)

\begin{tabular}{cc}
\hline Amostra & ASE $\left(\mathbf{m}^{2} / \mathbf{g}\right)$ \\
\hline Natural & 19,05 \\
Al-Fe-PILC & 173,49 \\
Pd-Al-Fe-PILC & 92,00 \\
\hline
\end{tabular}

As curvas termodiferenciais da bentonita natural e das amostras modificadas são mostradas na figura 2 . $\mathrm{Na}$ amostra natural, a perda de massa endotérmica em $101,2^{\circ} \mathrm{C}$ 
corresponde à perda de umidade, enquanto a perda de massa endotérmica em $714,3^{\circ} \mathrm{C}$ corresponde a desidroxilação de esmectita pobre em ferro ocataédrico, representando um colapso parcial da estrutura. Após essa temperatura, certamente o material perde parte de suas propriedades peculiares e suas aplicações catalíticas ficam comprometidas. Na região entre 800 e $1000^{\circ} \mathrm{C}$, há a presença do pico duplo endo-exotérmico. O primeiro (endotérmico) corresponde à destruição total do retículo cristalino da esmectita e o segundo (exotérmico) à formação de novas fases (Aranha, 2007). Após o processo de pilarização e a impregnação de $\mathrm{Pd}$, não se observaram grandes mudanças nos picos, porém existem certos deslocamentos na posição dos mesmos. O pico endotérmico em $714,3^{\circ} \mathrm{C}$ na bentonita natural apareceu em torno de $678^{\circ} \mathrm{C}$ na Al-Fe-PILC e Pd-Al-Fe-PILC. Apesar dessa ligeira diminuição da temperatura de desidroxilação, as amostras modificadas continuam apresentando elevada estabilidade térmica, propriedade fundamental para a catálise.

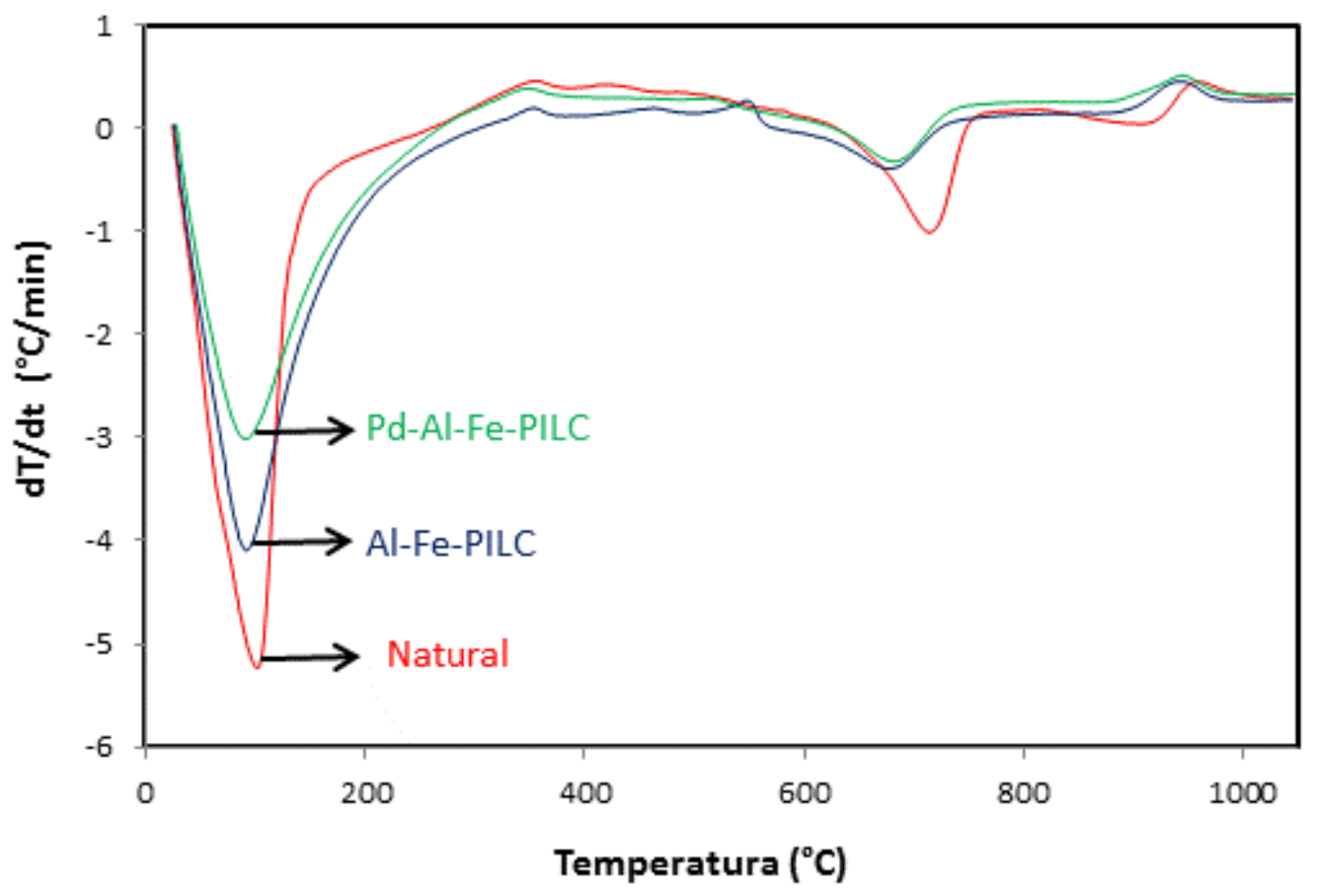

Figura 2 - Curvas termodiferenciais da amostra natural, Al-Fe-PILC e Pd-Al-Fe-PILC

\section{CONCLUSÃO}

Os materiais sintetizados apresentaram grandes espaçamentos basais, elevadas áreas superficiais específicas e estabilidades térmicas consideráveis. Os melhores resultados foram para a Al-Fe-PILC, indicando que esse material possui grande potencial de aplicação como catalisador de reações de oxidação, por exemplo. A Pd-Al-Fe-PILC apresentou valor de ASE menor que a Al-Fe-PILC e pelo seu difratograma, pode-se inferir que boa parte dos seus pilares se encontra colapsados. Entretanto o espaçamento basal da Pd-Al-Fe-PILC continuou sendo elevado, assim como sua estabilidade térmica, indicando que esse material possivelmente também possui potencial de aplicação como catalisador de reações simultâneas 
de oxidação e hidrodescloração, por exemplo. Testes catalíticos devem ser realizados para confirmar esses potenciais.

\section{REFERENCIAS}

ÁlVAREZ, A.; MORENO, S.; MOLINA, R.; IVANOVA, S.; CENTENO, M. A.; ODRIOZOLA, J. A. Gold supported on pillared clays for CO oxidation reaction: Effect of the clay aggregate size. Applied Clay Science, v. 69, p. 22-29, 2012.

ARANHA, I. B. Preparação, caracterização e propriedades de argilas organofílicas. Tese (Doutorado em Ciências), Universidade Federal do Rio de Janeiro (UFRJ), 2007.

BANKOVIC, P.; MILUTINOVIC-NIKOLIC, A.; MOJOVIC, Z.; JOVIC-JOVICIC, N.; ZUNIC, M.; DONDUR, V.; JOVANOVIC, D. Al,Fe-pillared clays in catalytic decolorization of aqueous tartrazine solutions. Applied Clay Science, v. 58, p. 73-78, 2012.

CAÑIZARES, P.; VALVERDE, J. L.; SUN-KOU, M. R.; MOLINA, C. B. Synthesis and characterization of PILCs with single and mixed oxide pillars prepared from two different bentonites. A comparative study. Microporous and Mesoporous Materials, v. 29, p. 267-281, 1999.

CATRINESCU, C.; ARSENE, D.; APOPEI, P.; TEODOSIU, C. Degradation of 4chlorophenol from wastewater through heterogeneous Fenton and photo-Fenton process, catalyzed by Al-Fe PILC. Applied Clay Science, v. 58, p. 96-101, 2012.

DE STEFANIS, A.; CAFARELli, P.; GALlESE, P.; GALlESE, F.; BORSELlA, E.; NANA, A.; PEREZ, G. Catalytic pyrolysis of polyethylene: A comparison between pillared and restructured clays. Journal of Analytical and Applied Pyrolysis, v. 104, p. 479-484, 2013.

GALEANO, L. A.; GIL, A.; VICENTE, M. A. Effect of the atomic active metal ratio in $\mathrm{Al} / \mathrm{Fe}-, \mathrm{Al} / \mathrm{Cu}-$ and $\mathrm{Al} /(\mathrm{Fe}-\mathrm{Cu})$-intercalating solutions on the physicochemical properties and catalytic activity of pillared clays in the CWPO of methyl orange. Applied Catalysis B: Environmental, v. 100, p. 271-281, 2010.

KOOLI, F. Porous clay heterostructures $(\mathrm{PCHs})$ from $\mathrm{Al}_{13}$-intercalated and $\mathrm{Al}_{13}$-pillared montmorillonites: Properties and heptane hydro-isomerization catalytic activity. Microporous and Mesoporous Materials, v. 184, p. 184-192, 2014.

LEÓN, M. A. D.; SANTOS, C. D. L.; LATRÓNICA, L.; CESIO, A. M.; VOLZONE, C.; CASTIGLIONI, J.; SERGIO, M. High catalytic activity at low temperature in oxidative dehydrogenation of propane with $\mathrm{Cr}-\mathrm{Al}$ pillared clay. Chemical Engineering Journal, v. 241, p. 336-343, 2014.

LUNA, J. F.; SCHUCHARDT, U. Argilas pilarizadas - uma introdução. Química Nova, v. 22, p. 104-109, 1999.

MNASRI, S.; BESBES, N.; FRINI-SRASRA, N.; SRASRA, E. Étude de l'activité catalytique des argiles pontées aluminium, zirconium et cérium dans la synthèse du 2,2-diméthyl-1,3dioxolane. Comptes Rendus Chimie, v. 15, p. 437-443, 2012.

MNASRI-GHNIMI, S.; FRINI-SRASRA, N. Promoting effect of cerium on the characteristic and catalytic activity of $\mathrm{Al}, \mathrm{Zr}$, and $\mathrm{Al}-\mathrm{Zr}$ pillared clay. Applied Clay Science, v. 88-89, p. 214-220, 2014. 
MOLINA, C. B.; CASAS, J. A.; ZAZO, J. A.; RODRÍGUEZ, J. J. A comparison of Al-Fe and $\mathrm{Zr}$-Fe pillared clays for catalytic wet peroxide oxidation. Chemical Engineering Journal, v. 118, p. 29-35, 2006.

MOLINA, C. B.; CALVO, L.; GILARRANZ, M. A.; CASAS, J. A.; RODRIGUEZ, J. J. PdAl pillared clays as catalysts for the hydrodechlorination of 4-chlorophenol in aqueous phase. Journal of Hazardous Materials, v. 172, p. 214-223, 2009.

MOLINA, C. B.; PIZARRO, A. H.; CASAS, J. A.; RODRIGUEZ, J. J. Aqueous-phase hydrodechlorination of chlorophenols with pillared clays-supported $\mathrm{Pt}, \mathrm{Pd}$ and $\mathrm{Rh}$ catalysts. Applied Catalysis B: Environmental, v. 148-149, p. 330-338, 2014.

MUNOZ, M.; PEDRO, Z. M.; CASAS, J. A.; RODRÍGUEZ, J. J. Chlorophenols breakdown by a sequential hydrodechlorination-oxidation treatment with a magnetic $\mathrm{Pd}-\mathrm{Fe} / \gamma-\mathrm{Al}_{2} \mathrm{O}_{3}$ catalyst. Water Reserach, v. 47, p. 3070-3080, 2013.

OLIVEIRA, L. C. A.; LAGO, R. M.; FABRIS, J. D.; SAPAG, K. Catalytic oxidation of aromatic VOCs with Cr or Pd-impregnated Al-pillared bentonite: Byproduct formation and deactivation studies. Applied Clay Science, v. 39, p. 218-222, 2008.

SILVA, M. V. C.; PIZARRO, A. H.; MOLINA, C. B.; ZAMIAN, J. R.; CARVALHO, S. M. L. Remoção de 4-clorofenol de soluções aquosas empregando argilas pilarizadas: ação combinada de Hidrodescloração catalítica e Oxidação catalítica com peróxido de hidrogênio. Scientia Plena, v. 10, n. 1, p. 1-12, 2014.

TEIXEIRA-NETO, E.; TEIXEIRA-NETO, A. M. Modificação química de argilas: Desafios científicos e tecnológicos para obtenção de novos produtos com maior valor agregado. Química Nova, v. 32, p. 809-817, 2009.

TOMUL, F. Adsorption and catalytic properties of Fe/Cr-pillared bentonites. Chemical Engineering Journal, v. 185-186, p. 380-390, 2012.

VICENTE, M. A.; LAMBERT, J. F. Synthesis of Pt pillared clay nanocomposite catalysts from $\left[\mathrm{Pt}\left(\mathrm{NH}_{3}\right)_{4}\right] \mathrm{Cl}_{2}$ precursor. Physical Chemistry Chemical Physics, v. 3, p. 4843-4852, 2001.

YALFANI, M. S.; GEORGI, A.; CONTRERAS, S.; MEDINA, F.; KOPINKE, F. Chlorophenol degradation using a one-pot reduction-oxidation process. Applied Catalysis B: Environmental, v. 104, p. 161-168, 2011. 